\title{
Batch Scheduling with Deteriorating Jobs to Minimize the Total Completion Time
}

\author{
Cuixia Miao • Yunjie Xia • Yuzhong Zhang • \\ Juan Zou
}

Received: 25 January 2013 / Revised: 26 June 2013 / Accepted: 17 July 2013 /

Published online: 30 August 2013

(C) Operations Research Society of China, Periodicals Agency of Shanghai University, and

Springer-Verlag Berlin Heidelberg 2013

\begin{abstract}
We consider bounded parallel-batch scheduling with proportional-linear deteriorating jobs and the objective to minimize the total completion time. We give some properties of optimal schedules for the problem and present for it a dynamic programming algorithm running in $O\left(b^{2} m^{2} 2^{m}\right)$ time, where $b$ is the size of a batch and $m$ is the number of distinct deterioration rates.
\end{abstract}

Keywords Batch scheduling · Deteriorating jobs · Dynamic programming algorithm

\section{Introduction}

The batch scheduling is motivated by burn-in operations in semiconductor manufacturing. Brucker et al. [1] said that a parallel-batch machine is a machine that can

This work was supported by the National Natural Science Foundation of China (Nos. 11201259, 11071142, 71101081), the Doctoral Fund of the Ministry of Education (Nos. 20123705120001, 20123705120003), the Natural Science Foundation of Shandong Province (Nos. ZR2011AL017, ZR2010AM034), Doctoral Research Fund (No. 20110130) and Postdoctoral Researcher of Qufu Normal University.

C. Miao · J. Zou

School of Mathematical Sciences, Qufu Normal University, Qufu, Shandong, 273165, P.R. China

C. Miao $(\bowtie)$

School of Physics and Engineering, Qufu Normal University, Qufu, Shandong, 273165, P.R. China e-mail: miaocuixia@126.com

Y. Xia

School of Office, Qufu Normal University, Qufu, Shandong, 273165, P.R. China

Y. Zhang $\cdot$ J. Zou

School of Management Sciences, Qufu Normal University, Rizhao, Shandong, 276826, P.R. China 
process up to $b$ jobs simultaneously as a batch, and the processing time of the batch is equal to the longest time of any job in the batch. All jobs contained in the same batch start and complete at the same time. Once processing of a batch is initiated, it cannot be interrupted and other jobs cannot be introduced into the batch until processing is completed. For the batch scheduling, there are two distinct models: the bounded model (i.e., $b<n$ ) and the unbounded model (i.e., $b \geqslant n$ ). This processing system has been extensively studied in the last two decades. Potts and Kovalyov [6] gave a survey on this scheduling model. Yuan et al. [8] gave some new results for the parallel-batch scheduling.

Traditional scheduling problems all assume that the processing times of jobs are constant. However, the processing times may change in the real world. Examples can be found in steel production and fire fighting, et al., where any delay in processing a task may increase its completion time. The monograph by Gawiejnowicz [2] presents this scheduling from different perspectives and covers results and examples. Liu et al. [3] gave some new results for this scheduling.

But job deterioration and parallel-batch processing co-exist in many realistic scheduling situations. Examples can be found in steel production. Qi et al. [7] gave some results for the unbounded parallel-batch scheduling with simple deteriorating jobs. Both of Li et al. [4] and Miao et al. [5] considered the parallel-batch scheduling with simple deteriorating jobs to minimize the makespan.

In this paper, we consider the bounded parallel-batch scheduling with $p_{j}=$ $\alpha_{j}(A+D t)$ to minimize the total completion time. Motivated by Brucker et al. [1], we present an $O\left(b^{2} m^{2} 2^{m}\right)$ time algorithm for the case with $m(<n)$ distinct deterioration rates.

\section{Problem Formulation}

The problem considered in this paper can be formally described as follows: there are $n$ independent non-preemptive deteriorating jobs $J=\left\{J_{1}, \cdots, J_{n}\right\}$ which are simultaneously available at time zero, to be scheduled on a single batch machine. Each job $J_{j}$ has a weight $w_{j}$ which is a measure of its importance. And the actual processing time of job $J_{j}$ is $p_{j}=\alpha_{j}(A+D t)$, where $\alpha_{j}$ be defined as the deterioration rate, $t$ the starting time, $A$ and $D$ are positive constant.

For a given batch $B$, we denote its deterioration rate and completion time by $\alpha(B)$ and $C(B)$, respectively. Define $\alpha(B)=\max \left\{\alpha_{j} \mid J_{j} \in B\right\}$. Following Gawiejnowicz [2], we denote our problem as $1 \mid p-$ batch, $p_{j}=\alpha_{j}(A+D t), b<n \mid \sum C_{j}$, where "p-batch" means parallel-batch.

A batch is full if it contains exactly $b$ jobs, otherwise it is non-full. If batch $B_{j}$ is sequenced after batch $B_{i}$ and $\alpha\left(B_{j}\right)<\alpha\left(B_{i}\right)$, then we say that $B_{j}$ is deferred with respect to $B_{i}$.

\section{Transformation Definition}

Lemma 3.1 ([2]) The problem $1\left|p_{j}=\alpha_{j}(A+D t)\right| C_{\max }$ is solvable in $O(n)$ time if $A, D>0, \alpha_{j}>0$ for $1 \leqslant j \leqslant n$, and the completion time of the $j$ th job and the 
maximum completion time are $C_{j}=\frac{A}{D} \prod_{i=1}^{j}\left(1+D \alpha_{i}\right)-\frac{A}{D}$ and $C_{\max }=\frac{A}{D} \prod_{j=1}^{n}(1+$ $\left.D \alpha_{j}\right)-\frac{A}{D}$, respectively.

Lemma 3.2 If $C_{j}^{\prime}=C_{j}+\frac{A}{D}$ for $1 \leqslant j \leqslant n$, where $C_{j}$ is the completion time of job $J_{j}$, then $C_{j}^{\prime}=C_{j-1}^{\prime}\left(1+D \alpha_{j}\right)$, where $C_{0}^{\prime}=\frac{A}{D}$.

Proof From the formula of $C_{j}$ in Lemma 3.1, we can prove this lemma.

Theorem 3.1 Problem $1 \mid p-$ batch, $p_{j}=\alpha_{j}(A+D t), b<n \mid \sum C_{j}$ is equivalent to problem $1 \mid p-$ batch, $p_{j}=\alpha_{j}(A+D t), b<n \mid \sum C_{j}^{\prime}$.

Proof We have $\sum_{j=1}^{n} C_{j}^{\prime}=\sum_{j=1}^{n}\left(C_{j}+\frac{A}{D}\right)=\sum_{j=1}^{n} C_{j}+\frac{n A}{D}$ since $C_{j}^{\prime}=C_{j}+\frac{A}{D}$, that is, the value of the new objective only increases the value of the original objective by $\frac{n A}{D}$, but it does not change the schedule. Thus, the two problems considered in this theorem is equivalent to each other since $A$ and $D$ are fixed. This completes the proof.

From Lemma 3.2, we know that the completion times of any two adjacent jobs are proportional in the new problem $1 \mid p-$ batch, $p_{j}=\alpha_{j}(A+D t), b<$ $n \mid \sum C_{j}^{\prime}$. Thus, the new problem is easier solvable than the original problem $1 \mid p-$ batch, $p_{j}=\alpha_{j}(A+D t), b<n \mid \sum C_{j}$. In the subsequent sections, combining with this proportional symbol, we design a dynamic programming algorithm for problem $1 \mid p-$ batch, $p_{j}=\alpha_{j}(A+D t), b<n \mid \sum C_{j}^{\prime}$. And then the original problem $1 \mid p-$ batch, $p_{j}=\alpha_{j}(A+D t), b<n \mid \sum C_{j}$ can be solved.

\section{Optimal Schedule Properties}

Suppose that we re-indexed the jobs in the non-decreasing order of their deterioration rates such that $\alpha_{1} \leqslant \cdots \leqslant \alpha_{n}$, i.e., the shortest deterioration rates order (SDR for short).

Theorem 4.1 There exists an optimal schedule for problem $1 \mid p-$ batch, $p_{j}=$ $\alpha_{j}(A+D t), b<n \mid \sum C_{j}^{\prime}$ in which each batch contains jobs with consecutive indices under the SDR order.

Proof Let $\pi=\left\{B_{1}, \cdots, B_{k}\right\}$ be any optimal schedule. Suppose that there are two batches $B_{x}, B_{y}$ with $x<y$ and three jobs $J_{j}, J_{j+1}, J_{j+2}$ with $\alpha_{j} \leqslant \alpha_{j+1} \leqslant \alpha_{j+2}$ such that $J_{j}, J_{j+2} \in B_{x}$ and $J_{j+1} \in B_{y}$ in schedule $\pi$. We get a new schedule $\pi^{\prime}$ by swapping job $J_{j+1}$ with $J_{j}$. Then, we have $\alpha\left(B_{x}\right)=\alpha\left(B_{x}^{\prime}\right), \alpha\left(B_{y}\right) \geqslant \alpha\left(B_{y}^{\prime}\right)$ and then $C_{\pi}^{\prime}\left(B_{x}\right)=S\left(B_{x}\right)\left(1+D \alpha\left(B_{x}\right)\right)=S\left(B_{x}\right)\left(1+D \alpha\left(B_{x}^{\prime}\right)\right)=C_{\pi^{\prime}}^{\prime}\left(B_{x}^{\prime}\right)$ and $C_{\pi}^{\prime}\left(B_{y}\right)=S\left(B_{y}\right)\left(1+D \alpha\left(B_{y}\right)\right) \geqslant S\left(B_{y}^{\prime}\right)\left(1+D \alpha\left(B_{y}^{\prime}\right)\right)=C_{\pi^{\prime}}^{\prime}\left(B_{y}^{\prime}\right)$. Thus, the total completion time for $\pi^{\prime}$ does not exceed that for $\pi$. Thus, the schedule $\pi^{\prime}$ is also optimal. A finite number of this procedure yields an optimal schedule of the required form. This completes the proof. 
From Lemma 2 and Theorem 6.197 in [2], we can get the following Lemma 4.1.

Lemma 4.1 For problem $1\left|p_{j}=\alpha_{j}(A+D t)\right| \sum w_{j} C_{j}^{\prime}$, it is optimal to sequence the jobs in the order $\frac{\alpha_{1}}{\left(1+D \alpha_{1}\right) w_{1}} \leqslant \cdots \leqslant \frac{\alpha_{n}}{\left(1+D \alpha_{n}\right) w_{n}}$.

It immediately leads to the subsequent theorem.

Theorem 4.2 There exists an optimal schedule $\left(B_{1}, \cdots, B_{k}\right)$ for problem $1 \mid p-$ batch, $p_{j}=\alpha_{j}(A+D t), b<n \mid \sum C_{j}^{\prime}$ with given batches $B_{1}, \cdots, B_{k}$ in which batches are scheduled in the order

$$
\frac{\alpha\left(B_{1}\right)}{\left(1+D \alpha\left(B_{1}\right)\right)\left|B_{1}\right|} \leqslant \frac{\alpha\left(B_{2}\right)}{\left(1+D \alpha\left(B_{2}\right)\right)\left|B_{2}\right|} \leqslant \cdots \leqslant \frac{\alpha\left(B_{k}\right)}{\left(1+D \alpha\left(B_{k}\right)\right)\left|B_{k}\right|} .
$$

Theorem 4.3 In any optimal schedule, there is no batch that is deferred with respect to a non-full batch.

The proof of this theorem is similar to that of Lemma 4 in [1].

\section{Scheduling with $m$ Distinct Deterioration Rates}

We present a backward dynamic programming algorithm in $O\left(b^{2} m^{2} 2^{m}\right)$ time for the case with $m(<n)$ distinct deterioration rates in this section.

Assuming that there are $m$ distinct deterioration rates, without loss of generality, let them be ordered by $\widetilde{\alpha_{1}} \leqslant \widetilde{\alpha_{2}} \leqslant \cdots \leqslant \widetilde{\alpha_{m}}$, and let $\widetilde{J_{i}}=\left\{J_{j} \mid \alpha_{j}=\widetilde{\alpha_{i}}\right\}$ for $i=1, \cdots, m$. Moreover, let $b_{i}=\left\lfloor\frac{\left|\widetilde{J}_{i}\right|}{b}\right\rfloor$.

We call a job is an $i$-job if its deterioration rate is $\widetilde{\alpha_{i}}$ and a batch is an $i$-batch if its longest job is $i$-job. We call an $i$-batch is pure if the batch is full and it contains $i$-job only, otherwise, a non-pure. The following theorem is similar to Lemma 6 in [1].

Theorem 5.1 There exists an optimal schedule satisfying the following properties: (i) there are $b_{i}$ pure $i$-batches and (ii) there is at most one non-pure $i$-batches for $i=1, \cdots, m$.

In the optimal schedule, there are $b_{i}$ pure $i$-batches. For the remaining jobs, we enumerate all possible configuration of full non-pure batches by Batch Filling Procedure [1]. We present a configuration of full non-pure batches by the set of induces $\Re \subset\{1, \cdots, m\}$, where $j \in \Re$ if and only if there is a full non-pure $j$-batch. We let

$$
a_{j, \Re}= \begin{cases}1 & \text { if } j \in \Re \\ 0 & \text { otherwise }\end{cases}
$$

be the number of full non-pure batch $j$-batch.

Now, we will form the non-full batches for the remaining jobs which have not been assigned by the batch filling procedure. Without loss of generality, let the remaining jobs be $J_{1}, \cdots, J_{n_{r}}$ with $\alpha_{1} \leqslant \cdots \leqslant \alpha_{n_{r}}$. We can draw the following conclusion, whose proof can be easily derived by Theorems 4.2 and 4.3 . 
Theorem 5.2 There exists an optimal schedule $\left(B_{1}, \cdots, B_{k}\right)$ for which under the SDR indexing order, i.e., $B_{l}=\left\{J_{j_{l}}, J_{j_{l}+1}, \cdots, J_{j_{l+1}-1}\right\}$ and $j_{l+1}-j_{l}<b$ for $l=$ $1, \cdots, k$, and where $1=j_{1}<\cdots<j_{l+1}=n_{r}+1$.

Now, we design an optimal schedule which has the following properties according to the above theorems:

$\diamond$ the full batches appear in order $\frac{\widetilde{\alpha_{1}}}{1+D \widetilde{\alpha_{1}}} \leqslant \cdots \leqslant \frac{\widetilde{\alpha_{m}}}{1+D \widetilde{\alpha_{m}}}$;

$\diamond$ the remaining jobs form non-full batches, where each such batch contains jobs with consecutive indices, and the batches are sequenced in SDR order;

$\diamond$ a complete schedule is found by interleaving the full and non-full batches.

For any $\Re$, we assume that the last remaining jobs have been indexed so that $\alpha_{1} \leqslant$ $\cdots \leqslant \alpha_{n_{r}}$.

Let $F_{i}(j)$ be the optimal value of the objective function satisfying the following conditions: the jobs in consideration are $J_{j}, \cdots, J_{n_{r}}$ which are assigned in non-full batches; the full batches in consideration are $k$-batches for $k=i, \cdots, m$.

To get $F_{i}(j)$, we take one of the following decisions in the previous state.

- Add all full $i$-batches and schedule them from time $\frac{A}{D}$.

The number of $i$-batches is $b_{i}+a_{i, \Re}$ and the deterioration rates are $\tilde{\alpha_{i}}$. Processing these batches in the schedule from time $\frac{A}{D}$, then there is a corresponding delay in the processing of all batches which have been assigned, and the completion time of jobs which already have been scheduled increase from $C_{j}^{\prime}$ to $\left(1+D \widetilde{\alpha_{i}}\right)^{b_{i}+a_{i, \Re}} C_{j}^{\prime}$. On the other hand, the total completion time of jobs containing in all full $i$-batches are

$$
\begin{aligned}
b & \frac{A}{D}\left[\left(1+D \widetilde{\alpha_{i}}\right)+\left(1+D \tilde{\alpha_{i}}\right)^{2}+\cdots+\left(1+D \widetilde{\alpha_{i}}\right)^{b_{i}+a_{i, \Re}}\right] \\
& =\frac{b A}{D^{2}} \frac{1+D \tilde{\alpha_{i}}}{\widetilde{\alpha_{i}}}\left[\left(1+D \tilde{\alpha_{i}}\right)^{b_{i}+a_{i, \Re}}-1\right] .
\end{aligned}
$$

Thus, $F_{i}(j)=\frac{b A}{D^{2}} \frac{1+D \widetilde{\alpha_{i}}}{\widetilde{\alpha_{i}}}\left[\left(1+D \widetilde{\alpha_{i}}\right)^{b_{i}+a_{i, \Re}}-1\right]+\left(1+D \widetilde{\alpha_{i}}\right)^{b_{i}+a_{i, \Re}} F_{i+1}(j)$.

- Add a non-full batch containing job $J_{j}$ and schedule it from time $\frac{A}{D}$.

Suppose that a batch be $\left\{J_{j}, \cdots, J_{k-1}\right\}$ which has deterioration rate $\alpha_{k-1}$, is inserted at the start of the schedule. The total completion time of these jobs is $\frac{A}{D}\left(1+D \alpha_{k-1}\right)(k-j)$ since $C_{0}^{\prime}=\frac{A}{D}$, and the completion times of assigned jobs increase from $C_{j}^{\prime}$ to $\left(1+D \alpha_{k-1}\right) C_{j}^{\prime}$. Thus, $F_{i}(j)=\left(1+D \alpha_{k-1}\right)\left[\frac{A}{D}(k-j)+\right.$ $\left.F_{i}(k)\right]$.

Combining the above two cases, we design a backward dynamic programming algorithm for any given $\Re$ as follows.

\section{Algorithm DPA}

Step 1 (Initialization) $F_{m+1}\left(n_{r}+1\right)=\frac{A}{D}$. 
Step 2 (Iteration) For $i=m+1, m, \cdots, 1$ and $j=n_{r}+1, n_{r}, \cdots, 1$ (where $i<$ $m+1$ or $\left.j<n_{r}+1\right)$

$$
F_{i}(j)=\min \left\{\begin{array}{l}
\frac{b A}{D^{2}} \frac{1+D \widetilde{\alpha_{i}}}{\widetilde{\alpha_{i}}}\left[\left(1+D \tilde{\alpha_{i}}\right)^{b_{i}+a_{i, \Re}}-1\right]+\left(1+D \widetilde{\alpha_{i}}\right)^{b_{i}+a_{i, \Re}} F_{i+1}(j) \\
\min _{j<k<\min \left\{j+b-1, n_{r}+1\right\}}\left\{\left(1+D \alpha_{k-1}\right)\left[\frac{A}{D}(k-j)+F_{i}(k)\right]\right\} .
\end{array}\right.
$$

\section{Step 3 (Solution)}

Define the optimal value $F^{*}=F_{1}(1)$.

Theorem 5.3 Problem $1 \mid p-$ batch, $p_{j}=\alpha_{j}(A+D t), b<n \mid \sum C_{j}^{\prime}$ can be solved in $O\left(b^{2} m^{2} 2^{m}\right)$ time for the case with $m(<n)$ distinct deterioration rates.

Proof For any given $\Re$, the recursion of $F_{i}(j)$ requires $O\left(m^{2} b^{2}\right)$ since the upper bound of the stored for $i=1, \cdots, m$ is $O(m)$ and the number of a batch is at most $b$ jobs and $n_{r} \leqslant m(b-1)$. There are at most $2^{m}$ possible combinations for the set of indices $\Re \subset\{1, \cdots, m\}$ since a $k$-job $(k=1, \cdots, m)$ belongs to a full non-pure batch or does not. Thus, $1 \mid p-$ batch, $p_{j}=\alpha_{j}(A+D t), b<n \mid \sum C_{j}^{\prime}$ can be solved in $O\left(b^{2} m^{2} 2^{m}\right)$. This completes the proof.

Now we have the following main result combing with Theorem 3.1.

Theorem 5.4 Problem $1 \mid p-$ batch, $p_{j}=\alpha_{j}(A+D t), b<n \mid \sum C_{j}$ can be solved in $O\left(b^{2} m^{2} 2^{m}\right)$ time for the case with $m(<n)$ distinct deterioration rates.

Accordingly, we conclude that the case with $m(<n)$ distinct deterioration rates of our problem can be solved in polynomial time when $m$ is fixed.

\section{Conclusions}

In this paper, we considered the bounded parallel-batch scheduling with proportionallinear deteriorating jobs to minimize the total completion time, and we presented a polynomial time dynamic programming algorithm when the number of distinct deterioration rates $m$ is fixed.

For future research, it would be worth considering the scheduling with the general deterioration type. Analysis of other objectives is another worthy topic.

Acknowledgements We thank the editor and the anonymous reviewers for their helpful and detailed comments on an earlier version of our paper.

\section{References}

[1] Brucker, P., Gladky, A., Hoogeveen, H., Kovalyov, M.Y., Potts, C.N., Tautenhahn, T., van de Velde, S.: Scheduling a batching machine. J. Sched. 1, 31-54 (1998)

[2] Gawiejnowicz, S.: Time-Dependent Scheduling, Monographs in Theoretical Computer Science. An EATCS Series, vol. 18. Springer, Berlin (2008) 
[3] Liu, M., Zheng, F.F., Chu, C.B., Zhang, J.T.: An FPTAS for uniform machine scheduling to minimize makespan with linear deterioration. J. Comb. Optim. 23, 483-492 (2012)

[4] Li, S.S., Ng, C.T., Cheng, T.C.E., Yuan, J.J.: Parallel-batch scheduling of deteriorating jobs with release dates to minimize the makespan. Eur. J. Oper. Res. 210, 482-488 (2011)

[5] Miao, C.X., Zhang, Y.Z., Cao, Z.G.: Bounded parallel-batch scheduling on single and multi machines for deteriorating jobs. Inf. Process. Lett. 111, 798-803 (2011)

[6] Potts, C.N., Kovalyov, M.Y.: Scheduling with batching: a review. Eur. J. Oper. Res. 120, 228-249 (2000)

[7] Qi, X.L., Zhou, S.G., Yuan, J.J.: Single machine parallel-btach scheduling with deteriorating jobs. Theor. Comput. Sci. 410, 830-836 (2009)

[8] Yuan, J.J., Qi, X.L., Lu, L.F., Li, W.H.: Single machine unbounded parallel-batch scheduling. Eur. J. Oper. Res. 186, 112-117 (2008) 